\section{Conventional Radio Frequency Thermo Coagulation versus Local Anesthetic and Steroid Injection for Ganglion Impar Block in Chronic Perineal Pain of Non-oncological Origin}

\author{
Hammad Usmani ${ }^{1 *}$, Roshan Andleeb ${ }^{1}$, Nazia Tauhee ${ }^{1}$, \\ Shaheen Anjum ${ }^{2}$ and Nayyar Asif ${ }^{3}$
}

${ }^{1}$ Department of Anesthesiology, Jawaharlal Nehru Medical College Hospital, Aligarh Muslim University, Aligarh, India

${ }^{2}$ Department of Obstetrics and Gynecology, Jawaharlal Nehru Medical College Hospital, Aligarh Muslim University, Aligarh, India

${ }^{3}$ Department of Orthopedic Surgery, Jawaharlal Nehru Medical College Hospital, Aligarh Muslim University, Aligarh, India

\begin{abstract}
Background: Chronic non-oncological perineal pain in female population is a well recognized medical condition. Chronic perineal pain of malignant or non malignant origin has been effectively managed by ganglion Impar block. Local anesthetic with or without steroid, phenol, alcohol, cryoablation and radiofrequency ablation are the accepted methods of blockade.

Objective: To compare the efficacy of local anesthetic-steroid combination and Conventional Radiofrequency (CRF) thermocoagulation for ganglion Impar block in female patients of chronic non-oncological perineal pain.

Methods: After informed and written consent; a prospective, randomized, double blind study was conducted on 70 female patients suffering from chronic perineal pain of non-malignant origin. The patients were divided into two groups of 35 each. In group CRF $(n=35)$, conventional radio frequency ablation was done and in group LAS $(n=35)$, local anaesthetic-steroid combination was used for ganglion Impar blockade. The extent of pain relief was assessed by Visual Analogue Scale (VAS) at $1^{\text {st }}$ week, $3^{\text {rd }}$ week, $6^{\text {th }}$ week
\end{abstract}

*Corresponding author: Hammad Usmani, Associate Professor, Department of Anesthesiology, Jawaharlal Nehru Medical College Hospital, Aligarh Muslim University, Aligarh, India, Tel: +91-9897114190; E-mail: hammadusmani2002@gmail.com

Citation: Usmani H, Andleeb R, Tauhee N, Anjum S, Asif N (2014 Conventional Radio Frequency Thermo Coagulation versus Local Anesthetic and Steroid Injection for Ganglion Impar Block in Chronic Perineal Pain of Non-Oncological Origin. J Anesth Clin Care 1: 005.

Received: July 22, 2014; Accepted: November 28, 2014; Published: December 11,2014 and $12^{\text {th }}$ week following the intervention. A questionnaire to evaluate subjective patient satisfaction was also used at each follow up visit.

Results: In group CRF, mean VAS score decreased significantly from the baseline value at each follow up visit; but in group LAS, this decrease was insignificant except at $1^{\text {st }}$ and $3^{\text {rd }}$ week follow up. Inter group comparison also showed significantly better pain relief in group CRF as compared to group LAS. At the end of $12^{\text {th }}$ week, 28 patients $(82 \%)$ in group CRF and 4 patients (12\%) patients in group LAS had excellent results as assessed by the subjective patient satisfaction questionnaire. Except infection at the site of skin puncture in a few patients, no other complication was seen in either group.

Conclusion: Ganglion Impar block by conventional radiofrequency provided significantly better pain relief with no major side effect in patients of chronic non-oncological perineal pain as compared to local anesthetic-steroid combination.

Limitations: Short term follow up period of only 12 weeks was a major drawback associated with this study.

Keywords: Chronic non-oncological perineal pain; Conventional radiofrequency thermocoagulation; Corticosteroid; Female; Ganglion impar block; Local anesthetic

\section{Introduction}

Chronic perineal pain is an idiopathic multi factorial vague disorder which results due to a complex interaction between neurological, musculoskeletal and endocrine systems. The behavioural and psychological factors play an important role in the persistence of this pain syndrome $[1,2]$.

The ganglion Impar (ganglion of Walther) is a single retroperitoneal bundle of nerves located near the sacrococcygeal joint and marks the end of two paravertebral sacral sympathetic chains [3]. Blockade of the ganglion Impar has been widely accepted intervention for the management of recalcitrant chronic perineal pain of visceral and/or sympathetic origin [4-6].

Blockade of ganglion Impar is usually done by injection of local anesthetic with or without steroids $[4,7,8]$, phenol or alcohol $[3,4,9]$, botox [6] or cryoablation [10] through various approaches like anococcygeal [4], trans-sacrococcygeal [11-13], trans-coccygeal [14-17]. Latest addition to this list is a non-pharmacological technique of Conventional Radiofrequency Ablation (CRF). In CRF application, $420 \mathrm{kHz}$ high frequency alternating current at a voltage output of 15-25 volt is applied for 60 to 90 seconds, generating a temperature of $60^{\circ} \mathrm{C}$ to $80^{\circ} \mathrm{C}$. This temperature causes selective thermocoagulation of pain carrying nerve fibers (A- $\delta$ and $C$ fibers) [18-20]. It has got an additional advantage of creating a well circumscribed lesion, thus sparing the adjacent vital structures.

Recently, few clinical trials were conducted to study the efficacy of CRF of ganglion Impar block for the management of chronic perineal pain $[3,21,22]$ but none of the authors have compared the efficacy of local anaesthetic-steroid combination with CRF for chronic perineal pain. 
In the present study, the efficacy of local anesthetic-steroid combination was compared with CRF for ganglion Impar block in 70 female patients of chronic perineal pain of non-oncological origin.

\section{Methods}

This was a prospective, randomized, double blind study conducted at Pain Clinic, Department of Anesthesiology, Jawaharlal Nehru Medical College Hospital, Aligarh Muslim University, Aligarh from February 2011 to March 2014. A due approval from the ethics committee of the institution was obtained for the study.

\section{Participants}

After taking written and informed consent, 70 female patients aged between 15-75 years with chronic non-oncological perineal pain of at least six weeks duration, unresponsive to conservative treatment were selected. Diagnostic ganglion Impar block with $0.25 \%$ bupivacaine was performed and the patients showing at least $50 \%$ pain relief lasting for 2-6 hours were enrolled for the study. Patients with coexisting coagulation abnormalities or prolonged bleeding time, septicemia as demonstrated by elevated white blood cells and infection overlying the entry area were excluded from the study. Patients receiving anticoagulants/aspirin containing NSAID and showing hypersensitivity to local anesthetic agents were also excluded.

\section{Randomization}

Patients were randomly divided into two groups of 35 patients each, using computer generated randomization schedule. Group CRF $(\mathrm{n}=35)$ underwent conventional radiofrequency thermocoagulation and group LAS $(\mathrm{n}=35)$ was treated with local anaesthetic-steroid combination containing $0.25 \%$ bupivacaine and methylprednisolone $60 \mathrm{mg}$.

\section{Interventions}

The procedure was performed with the patient in the prone position on an x-ray translucent table. C-arm (Multimobil 5C, Siemens Ltd, India) was used to identify and mark the essential bony landmarks. No sedation was given. Under strict aseptic precautions, sacral and gluteal part was cleaned and draped and access region was left open. In order to view the first intra-coccygeal joint, C-arm was taken to lateral position. After infiltrating the skin and subcutaneous tissues with 2\% lidocaine; an 18G, 1.5 inch hypodermic needle was passed through the first intra-coccygeal joint perpendicularly and advanced till it pierced the deep dorsal intracoccygeal ligament. Through this introducer needle, a $10 \mathrm{~cm}$ long $22 \mathrm{G}$ radiofrequency cannula with $5 \mathrm{~mm}$ active tip (Cosman Medical) was passed, till the tip reached anterior to ventral intra-coccygeal ligament, felt as loss of resistance. Position of needle tip was confirmed by injecting $1 \mathrm{ml}$ of radio-opaque dye in the retroperitoneal space. The spread of dye gives a reverse comma appearance in the lateral view (Figure1). Through this radiofrequency cannula, radio frequency thermocouple electrode (Cosman Medical) was passed. Before radiofrequency ablation, tissue impedance check, motor and sensory tests were performed, which comprised of tissue impedance $<600 \mathrm{Ohm}$, sensory response below $50 \mathrm{~Hz}$ and a motor response below $2 \mathrm{~Hz}$. Cosman Radiofrequency Generator (RFG-1A) was used in this study.

Patients of CRF group underwent radiofrequency lesioning at $80^{\circ} \mathrm{C}$ for 90 seconds while in LAS group, $3 \mathrm{ml}$ solution of $0.25 \%$ bupivacaine and methylprednisolone $60 \mathrm{mg}$ was injected through 22 gauge cannula.

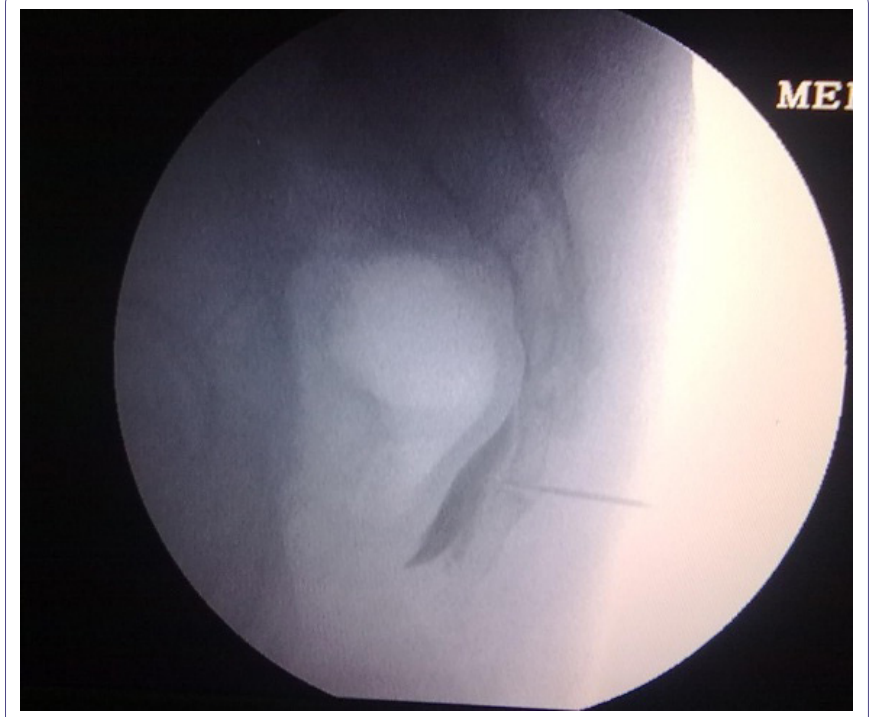

Figure 1: Dye spread as seen in ganglion Impar block under C-arm guidance.

After completion of the procedure, the patients were shifted to the recovery room, where they were monitored for 4 hours. Follow up of patients was done at $1^{\text {st }}$ week, $3^{\text {rd }}$ week, $6^{\text {th }}$ week and $12^{\text {th }}$ week through Pain Clinic visits. The patients were allowed to take the combination of Tramadol $37.5 \mathrm{mg}$ and Paracetamol $325 \mathrm{mg}$ in the tablet form as and when required during the study period with a minimum interval of 6 hours between two doses. Gabapentin however, was continued in the same doses as in the pre-treatment period.

\section{Outcome measures}

Pain intensity was measured in pre and post treatment period by $10 \mathrm{~cm}$ Visual Analogue Scale (VAS). In this scale, "0" indicates no pain whereas " 10 " indicates the worst imaginable pain. VAS scores at $1^{\text {st }}$ week, $3^{\text {rd }}$ week, $6^{\text {th }}$ week and $12^{\text {th }}$ week follow up visits were recorded. The investigator blinded to the study groups collected the data from the patients.

The patients were also asked to express their satisfaction through a set of questionnaire at each follow up visit (Table 1). Any complication occurred during the procedure or in follow up period was also noted.

\begin{tabular}{|c|c|}
\hline Measure & Outcome \\
\hline Significant pain relief and improvement in sitting and standing activity & Excellent \\
\hline Less pain but require intermittent analgesics & Good \\
\hline $\begin{array}{l}\text { Using the same analgesic as before ganglion Impar block, only mild or } \\
\text { no improvement }\end{array}$ & Poor \\
\hline
\end{tabular}

Table 1: Questionnaire for subjective evaluation of the patient.

\section{Sample size}

Sample size for the study was calculated using the Power and Sample size calculation program (version 3.0.43) and minimum number of patients required was found to be 31 with power of at least $80 \%$ and $\alpha=0.05$. Anticipating loss of subjects during the study, 35 patients were enrolled in each group.

\section{Statistical analysis}

Data were analyzed using the statistical package SPSS version 11.0 for Windows (SSPS Inc, Chicago, IL, USA). VAS scores were analyzed using student $\mathrm{t}$-tests and patient satisfaction was analyzed by using chi-square test. Results were presented as a mean \pm Standard 
Deviation (SD) and percentage. A p-value $<0.05$ was considered statistically significant.

\section{Results}

\section{Participant flow}

One patient in group CRF and two patients in group LAS did not turn up in the follow up period and therefore excluded from the study (Figure 2).

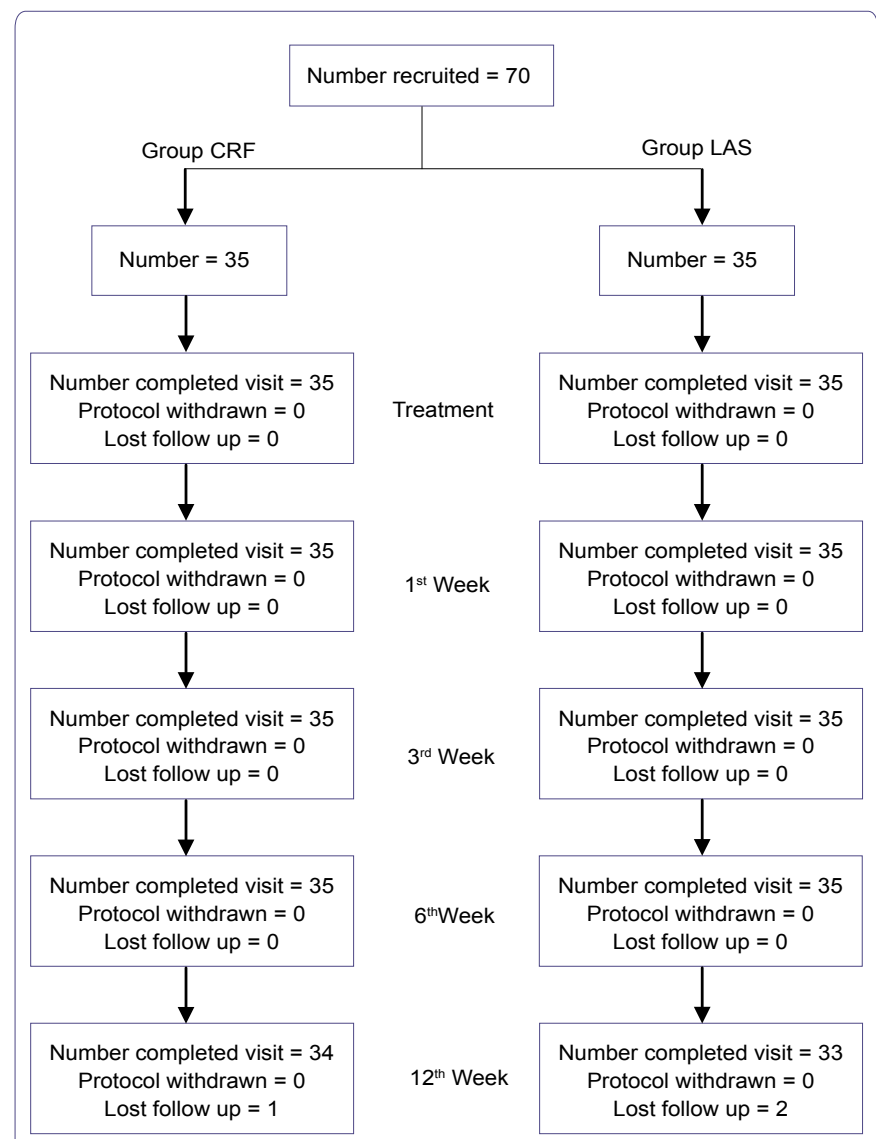

Figure 2: Study design and participant flowchart.

\section{Evaluation of demographic variables}

The study groups were comparable with respect to age and duration of pain (Table 2).

\begin{tabular}{|l|c|c|}
\hline Parameter & Group CRF $(\mathbf{n}=\mathbf{3 4})$ & Group LAS $(\mathbf{n = 3 3})$ \\
\hline Age (years) & $39.32 \pm 14.29$ & $42.32 \pm 15.64$ \\
\hline Duration of pain (months) & $13.7 \pm 9.3$ & $15.2 \pm 8.3$ \\
\hline Diagnosis: & & \\
Coccygodynia & $24(70.6 \%)$ & $23(69.7 \%)$ \\
Chronic anal pain & $03(8.8 \%)$ & $03(9.1 \%)$ \\
Post episiotomy chronic perineal pain & $04(11.8 \%)$ & $05(15.1 \%)$ \\
Sacrococcygeal pain & $01(2.9 \%)$ & $00(0 \%)$ \\
Vulvodynia & $02(5.9 \%)$ & $02(6.1 \%)$ \\
\hline
\end{tabular}

Table 2: Demographic profile of patients in both the study groups.

\section{Pain relief}

Patients of both the groups reported significant pain relief at the time of discharge i.e. 4 hours after the block. The extent of pain relief was more or less similar at $1^{\text {st }}$ and $3^{\text {rd }}$ week in both the study groups; however, at sixth and twelfth week of the study, patients in group CRF showed significantly better pain relief as compared with patients in group LAS (Figure 3 and Table 3 ).

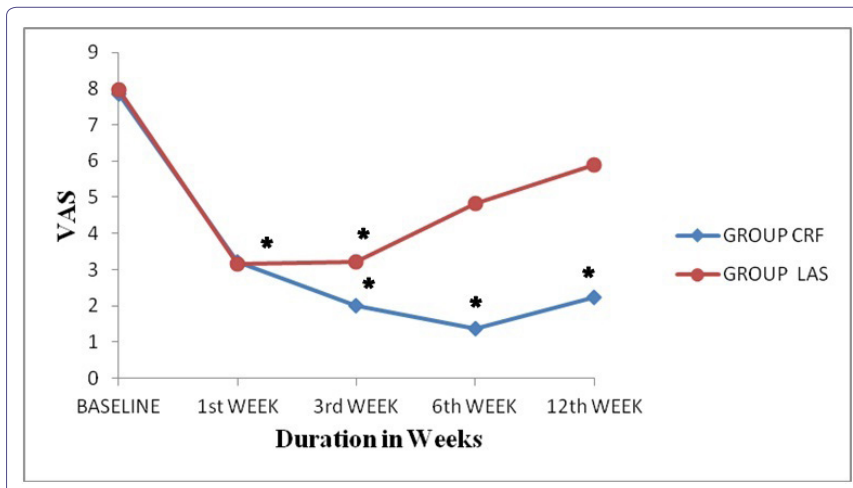

Figure 3: VAS scores in the two study groups.

$\mathrm{p}<0 .{ }^{*} 05$ from baseline value.

\begin{tabular}{|c|c|c|c|c|c|}
\hline & Group CRF & Group LAS & $\mathbf{P}^{*}$ & $\mathbf{P}^{\dagger}$ & $\mathbf{P}^{\S}$ \\
\hline Baseline & $7.84 \pm 1.28$ & $7.96 \pm 1.17$ & - & - & 0.4718 \\
\hline $1^{\text {st }}$ week & $3.20 \pm 0.76$ & $3.16 \pm 1.03$ & 0.0001 & 0.0006 & 0.3894 \\
\hline $3^{\text {rd }}$ week & $2.00 \pm 1.22$ & $3.20 \pm 0.71$ & 0.0001 & 0.0331 & 0.999 \\
\hline $6^{\text {th }}$ week & $1.36 \pm 1.11$ & $4.84 \pm 0.8$ & 0.0001 & 0.2514 & 0.0001 \\
\hline $12^{\text {th }}$ week & $2.24 \pm 1.56$ & $5.88 \pm 1.54$ & 0.0001 & 0.1793 & 0.0001 \\
\hline
\end{tabular}

Table 3: VAS scores in the study groups before and after treatment

*: Significance of group CRF from baseline value; $\dagger$ : Significance of group LAS from baseline; §: Significance between group CRF and group LAS

\section{Subjective evaluation of patient satisfaction}

Patient satisfaction was graded as excellent or good by a significantly large number of patients at $1^{\text {st }}, 3^{\text {rd }}, 6^{\text {th }}$ and $12^{\text {th }}$ week of study in group CRF as compared to group LAS (Table 4 and Figure 4).

\begin{tabular}{|l|c|c|c|}
\hline & Excellent + Good & Poor & *P-value \\
\hline $1^{\text {st }}$ week: & & & \\
Group CRF $(n=34)$ & $24(70.6 \%)$ & $10(29.4 \%)$ & 0.39 \\
Group LAS $(n=33)$ & $20(60.6 \%)$ & $13(39.4 \%)$ & \\
\hline $3^{\text {rd }}$ week: & & & \\
Group CRF $(n=34)$ & $26(76.5 \%)$ & $08(23.5 \%)$ & 0.16 \\
Group LAS $(n=33)$ & $18(54.5 \%)$ & $15(45.5 \%)$ & \\
\hline $6^{\text {th }}$ week: & & & \\
Group CRF $(n=34)$ & $28(88.2 \%)$ & $06(11.8 \%)$ & 0.00 \\
Group LAS $(n=33)$ & $10(21.2 \%)$ & $23(78.8 \%)$ & \\
\hline $12^{\text {th }}$ week: & & & \\
Group CRF $(n=34)$ & $28(82.3 \%)$ & $06(17.6 \%)$ & 0.00 \\
Group LAS $(n=33)$ & $04(12.1 \%)$ & $29(87.9 \%)$ & \\
\hline
\end{tabular}

Table 4: Evaluation of patient satisfaction in the two study groups.

*: Significance of patients showing excellent + good satisfaction in the two study goups.

\section{Adverse events}

Patients were discharged from the hospital after 4 hours of observation without any adverse sequelae. No untoward side effect or neurological complication other than infection at the site of skin puncture was observed during the follow up period in any of the patient of the two groups. Infection at the site of skin puncture was found in 3 out of 34 patients in group CRF and 2 out of 33 patients in group LAS, which resolved after oral antibiotic treatment. The incidence of this complication was comparable in both the study groups. 


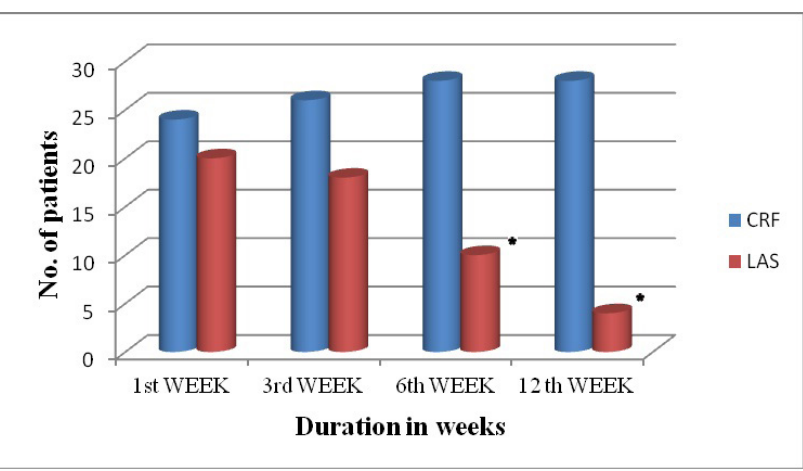

Figure 4: Patients showing excellent+good satisfaction during the study period.

*: Significance $(p<0.05)$ between group CRF versus group LAS

\section{Discussion}

A considerable number of studies are available to confirm the efficacy of local anaesthetic-steroid combination for ganglion Impar block. The relief obtained with local anaesthetic steroid combination varies in amount and duration from weeks to months. The procedure is of low risk and can be repeated several times to achieve optimal pain relief. Toshniwal et al., [11] conducted a prospective study on six patients who received ganglion Impar block with $0.25 \%$ bupivacaine and $40 \mathrm{mg}$ of methylprednisolone acetate for chronic perineal pain, through a trans-sacrococcygeal approach. All the patients had significant pain relief during two month follow up period, without any adverse events. Datir and Connell [23] performed CT-guided ganglion Impar blocks using a combination of bupivacaine and triamcinolone on eight patients suffering from coccydynia. Three patients (37\%) showed complete pain relief on all follow-up visits up to six months. Saenza et al., [24] carried out a retrospective observational study on 23 patients of chronic post-traumatic coccydynia. They were treated by radiologically guided infiltration of the ganglion Impar through the sacrococcygeal ligament or through the first intra-coccygeal junction with $1 \%$ lidocaine and $60-80 \mathrm{mg}$ triamcinolone. The effectiveness of the ganglion Impar block in reducing VAS scores by at least 50\% was reported in $60.94 \%$ (13 cases). 6 patients required repetition of the technique due to recurrence of pain six months after the block. The only side effect observed was pain at the puncture site for two to three days after the procedure.

Few clinical trials have recently been undertaken to study the efficacy of CRF of ganglion Impar block for the management of chronic perineal pain. Reig et al., [3] did a prospective study on 13 patients with chronic non-oncological perineal pain, who underwent $\mathrm{CRF}$ of ganglion Impar at $80^{\circ} \mathrm{C}$ for 80 seconds. VAS scores decreased significantly from baseline by an average of more than $35 \%$ in the whole group. The mean duration of improvement was 2.2 months (range: 1 to 6 months). Abejon et al., [21] conducted a retrospective observational study on 21 patients of chronic perineal pain of diverse etiology. Complete pain relief was achieved in three patients, more than $50 \%$ relief in 11 patients and less than the 50\% in remaining patients after CRF ablation of ganglion Impar. Demicray et al., [22] conducted a retrospective study on 10 patients with chronic coccydynia treated by $\mathrm{CRF}\left(80^{\circ} \mathrm{C}\right.$ for 120 seconds). Statistically significant differences were observed between the pre-procedure and post-procedure visual numeric pain scale score. Mid-term evaluation after the treatment ( 6 months) revealed that $90 \%$ of the patients had a successful outcome and $10 \%$ were deemed failures.

In the present study, the effectiveness of CRF can be observed from the significant differences in pre-treatment and post-treatment VAS scores and patient satisfaction during $12^{\text {th }}$ weeks of study period. Pain scores were significantly reduced from the baseline value in group CRF at $1^{\text {st }}, 3^{\text {rd }}, 6^{\text {th }}$ and $12^{\text {th }}$ week follow up visits, while reduction in VAS scores in group LAS was insignificant at $6^{\text {th }}$ and $12^{\text {th }}$ week. Analysis of patient satisfaction at the end of follow up period showed that $80 \%$ in group CRF had an excellent outcome as compared to only $12 \%$ patients of group LAS.

Ganglion Impar block may not be successful in all patients because of anatomical variations of the ganglion, possibly contributing to ineffective block. Oh et al., [25] studied the anatomical variability of the ganglion Impar and found that in $26 \%$ of the cadavers, it was located anterior to lower segment of first coccyx and in $18 \%$, it was anterior to sacro-coccygeal junction. Our approach to ganglion Impar was through the first intra-coccygeal joint and the procedure was carried out under fluoroscopic guidance.

Since CRF is a painful procedure, local anesthetic was given prior to its application. Post-procedure de-afferentation pain, neuritis or neurogenic inflammation, painful cutaneous dysthesias, allodynia or motor deficit may occur [18], but rarely. The only complication observed in our study was infection at the site of skin puncture, which was successfully treated with one week of oral antibiotic therapy.

\section{Limitations}

The main limitation of our study was the short term follow up period of only $12^{\text {th }}$ weeks. Another important limitation of our study was that the sample size of 35 was calculated using an $\alpha=0.05$ and power of study $80 \%$. Precision and accuracy could have been better had the sample size been calculated using a power of $90 \%$.

To conclude, our study revealed that the ganglion Impar block by conventional radiofrequency provided significantly better pain relief with no major side effect in female population suffering from chronic perineal pain of non oncological origin as compared to local anesthetic-steroid combination.

\section{References}

1. Wesselmann U, Burnett AL, Heinberg LJ (1997) The urogenital and rectal pain syndromes. Pain 73: 269-294.

2. Burnett AL, Wesselmann $U$ (1999) Neurobiology of the pelvis and perineum principles for a practical approach. J Pelvic Surg 5: 224-232.

3. Reig E, Abejón D, del Pozo C, Insausti J, Contreras R (2005) Thermocoagulation of the ganglion Impar or ganglion of Walther: description of a modified approach. Preliminary results in chronic, nononcological pain. Pain Pract 5 : 103-110.

4. Plancarte R, Amescua C, Patt RB, Silvia A (1990) Presacral Blockade of the Ganglion of Walther (Ganglion Impar). Anesthesiology 73: 751.

5. Bașağan M E, Türker G, Kelebek GN, Uçkunkaya N, Sahin S (2004) [Blockade of ganglion Impar through sacrococcygeal junction for cancer-related pelvic pain]. Agri 16: 48-53.

6. Lim SJ, Park HJ, Lee SH, Moon DE (2010) Ganglion Impar block with botulinum toxin type $\mathrm{A}$ for chronic perineal pain -A case report. Korean $\mathrm{J}$ Pain 23: 65-69.

7. Kabbara Al (2005) Transsacrococcygeal ganglion Impar block for postherpetic neuralgia. Anesthesiology 103: 211-212. 
8. McAllister RK, Carpentier BW, Malkuch G (2004) Sacral postherpetic neuralgia and successful treatment using a paramedial approach to the ganglion Impar. Anesthesiology 101: 1472-1474.

9. deLeón-Casasola OA (2000) Critical evaluation of chemical neurolysis of the sympathetic axis for cancer pain. Cancer Control 7: 142-148.

10. Loev MA, Varklet VL, Wilsey BL, Ferrante MF (1998) Cryoablation: A nove approach to neurolysis of the ganglion Impar. Anesthesiology 88: 1391-1393.

11. Toshniwal GR, Dureja GP, Prashanth SM (2007) Transsacrococcygeal Approach to Ganglion Impar block for Management of Chronic Perineal Pain: A Prospective Observational Study. Pain Phys 10: 661-666.

12. Eker HE, Cok OY, Kocum A, Acil M, Turkoz A (2008) Transsacrococcygeal approach to ganglion Impar for pelvic cancer pain: a report of 3 cases. Reg Anesth Pain Med 33: 381-382.

13. Kuthuru MR, Kabbara Al, Oldenburg PF, Boswell MV, Rosenberg SK (2003) Coccygeal pain relief after transsacrococcygeal block of the ganglion Impar under fluoroscopy: A case report. Arch Phys Med Rehabil 84: 24

14. Wemm JR, K, Saberski L (1995) Modified approach to block the ganglion Impar (ganglion of Walther). Reg Anesth 20: 544-545.

15. Foye PM (2007) New approaches to ganglion Impar blocks via coccygeal joints. Reg Anesth Pain Med 32: 269.

16. Hong JH, Jang HS (2006) Block of the ganglion Impar using a coccygeal joint approach. Reg Anesth Pain Med 31: 583-584.
17. Foye PM (2008) Ganglion Impar blocks via coccygeal versus sacrococcygeal joints. Reg Anesth Pain Med 33: 279-280.

18. Sluijter ME (2001) Radiofrequency: part 1. Flivopress Meggen, Switzerland.

19. Byrd D, Mackey S (2008) Pulsed radiofrequency for chronic pain. Curr Pain Headache Rep 12: 37-41.

20. Sluijter M, Racz G (2002) Technical aspects of radiofrequency lesioning. Pain Pract 2: 195-200.

21. Abejon D, Pcheco MD, Cortina I, Romero A, del Pozo C, et al. (2007) Treatment of perineal pain with thermocoagulation of the ganglion Impar. Rev Soc Esp dolor 4: 290-295.

22. Demircay E, Kabatas S, Cansever T, Yilmaz C, Tuncay C, et al. (2010) Radiofrequency thermocoagulation of ganglion Impar in the management of coccydynia: preliminary results. Turk Neurosurg 20: 328-333.

23. Datir A, Connell D (2010) CT-guided injection for ganglion Impar blockade: a radiological approach to the management of coccydynia. Clin Radiol 65: 21-25.

24. Sáenz MC, Cerrillo JAI, Colomo CV, Vélez GS, Pizarro AQ, et al. (2011) Infiltration of Impar and caudal ganglia in the management of traumatic coccydynia refractory to conventional analgesic treatment. Rev esp cir ortop traumatol 55: 340-345.

25. Oh CS, Chung IH, Ji HJ, Yoon DM (2004) Clinical implication of topographic anatomy on the ganglion Impar. Anesthesiology 101: 249-250. 